\title{
Characteristics of Residential Tracker Accuracy in Quantified Direct Beam Irradiance and Global Horizontal Irradiance
}

\author{
M.S. Sabry and B.W. Raichle
}

Department of Technology and Environmental Design, Appalachian State University, Boone, NC 28608, USA

\begin{abstract}
An accurate solar tracker matches array angles with solar angles throughout the day. Many studies have used the power produced by a tracked PV array as a proxy to characterize a tracker's accuracy. However, it is difficult to decouple the effects of tracker performance from other effects on power output. It was not found in the literature reviewed that there are studies that determine the accuracy of solar trackers by directly measuring the tracker angles. This study was an experiment to determine the accuracy of two small commercially available non-algorithm based solar trackers: the Zomeworks UTR-020 passive one axis solar tracker, and the Wattsun AZ-225 active electro-optical two axis solar tracker. Accuracy of the trackers was determined by measuring the tracking angles under varying conditions including direct beam irradiation ( $\mathrm{DBI}$ ) and global horizontal irradiance $(\mathrm{GHI})$, and comparing to calculated sun angles. The results showed that the average azimuth angle accuracy of the Zomeworks UTR-020 is $75 \%$, the average azimuth angle accuracy of the Wattsun AZ-225 is $88 \%$, and the average elevation angle accuracy of the Wattsun is $89 \%$. In addition, the results showed a weak correlation between the azimuth accuracy of the Zomeworks and DBF, a strong correlation between the azimuth accuracy of the Wattsun and DBF, and a moderate correlation between the elevation accuracy of the Wattsun and DBF. Moreover, the azimuth accuracy of the Wattsun was always higher than that of the Zomeworks under all DBF and GHI conditions.
\end{abstract}

Keywords: Solar Tracker, Accuracy, Tracking Error, Non Algorithm Based, Solar Tracking Angles.

\section{INTRODUCTION}

Interest in solar energy has grown for a variety of reasons. It is a sustainable energy resource, it produces no emissions, and it is increasingly affordable and reliable. Use of solar energy can help to mitigate greenhouse gas emissions, and therefore many policies have been developed and adopted, and many projects have been undertaken worldwide to produce solar energy and to reduce the production of greenhouse gases.

Photovoltaic (PV) technology has continued to develop in recent years. Tracking technology that maximizes the power output of PV panels is just one area of improvement. The increase in power generated from a PV array mounted on a solar tracker is due to an increase of the received irradiation. "The surplus of irradiance received by one-axis tracking and two-axis tracking systems were $34 \%$ and $38 \%$ " [1]. In addition, "tests have shown that up to $40 \%$ extra power can be produced per annum using a variable elevation solar tracker" [2].

To maximize radiation hitting the surface of a fixed PV array throughout the year, the array needs to be adjusted to certain elevation and azimuth angles. Better performance can be obtained if an array tracks the sun throughout the day by continually adjusting its azimuth and/or elevation angles. By adjusting these

*Address correspondence to this author at the Department of Technology and Environmental Design, Appalachian State University, Boone 28608, USA, “Attn: BW Raichle.”; Tel: (+20) 106-965-2078; E-mail: sabryms@appstate.edu angles, the direct solar radiation is more normal to the array's surface throughout the day; the array effective area is increased, and thus better energy output will result.

Tracking accuracy refers to the ability of a tracker to point to the sun. To achieve high accuracy, the tracking system must accurately know where the sun is. Knowing the tracking accuracy will be helpful to potential adopters of $\mathrm{PV}$ tracking systems.

A variety of tracking control schemes exists, including algorithm-based, thermal, and electro-optical. An algorithm-based control scheme provides the equations through which the elevation and azimuth angles are calculated and employs active positioning using electric motors. This guarantees the optimum orientation of the trackers. Non-algorithm based control schemes, such as thermal and electro-optical, rely on sensing some parameter that varies with solar position. These systems likely are less accurate under non-ideal radiation conditions. Most residential-scale solar trackers are non-algorithm based. It is important to understand the performance of these trackers and to determine their accuracy.

\section{LITERATURE REVIEW}

\subsection{Solar Irradiation and Meteorological Conditions}

As solar radiation passes through the atmosphere it interacts with air molecules and particulates in the atmosphere and thus the ray's angle changes, making 
the solar rays diffuse rather than direct [3]. If the weather is windy, cloudy, rainy, or snowy, there will be more scattering of solar rays. Direct Normal Irradiance DNI (also known as Direct Beam Irradiance DBI) is "the radiation that is not reflected or scattered and reaches the surface directly" [1]. Diffuse radiation is "radiation that has been scattered either by clouds, rain, or any other potential hazard" [3]. The amount of direct or diffuse radiation depends on the position of the sun, atmospheric conditions, and the orientation of the receiving surface. The percentage of direct beam radiation from the total irradiance is known as Direct Beam Fraction DBF [3].

\subsection{Solar Tracking Geometry}

Understanding the relationship between sun angles and the orientation angles of the PV array's surface is fundamental to determining tracker accuracy. It is important to know surface azimuth and surface elevation angles as well as solar azimuth and solar elevation angles.

Figure 1 shows angle definitions. The surface azimuth angle $\alpha_{p}$ is the angle between north and the projection of the surface normal in the horizontal plane. The surface elevation angle $\gamma_{\mathrm{p}}$ is the vertical angle between the surface and the horizontal plane (or between the surface normal and the zenith). The solar azimuth angle $\alpha_{\mathrm{s}}$ is the angle between north and the projection of the sun's ray in the horizontal plane. The solar elevation angle $\gamma_{s}$ is the vertical angle between the horizontal plane and the sun's rays. The incident angle, $\theta$, is measured between the sun's direct rays and the surface normal.

In order to have an optimum orientation, the tracker would consistently point the array such that the surface normal is parallel to the direct incident solar radiation.

\subsection{Solar Tracking Technologies}

Solar tracking technologies are usually classified into passive (mechanical) or active (electrical) devices. They can be further classified into one axis solar trackers or dual axis solar trackers, as shown in Figure 2. The solar trackers used in the study are the Zomeworks UTR-020 and the Wattsun AZ-225.

The Zomeworks UTR-020 is a small one axis passive solar tracker. The array rotates in azimuth about a fixed, tilted elevation axis - in this case tilted at $40^{\circ}$. Rotation is caused by a difference in weight of two connected tubes filled with a working fluid (Freon). Aluminum shadow plates result in differential solar heating of the tubes.

In the morning, the Zomeworks begins its motion from where it stopped at sunset of the previous day. As the sun rises, as in Figure 3, its rays hit one of the tubes causing the working fluid to expand and travel to the other, shaded tube. The shaded tube is now heavier and the tracker rotates to balance the torques. Equilibrium is reached when the sun's rays are normal to the surface of the array placing each tube in equal shading. This behavior takes place throughout the day until sunset.

The Wattsun AZ-225 is a two axis active solar tracker. Azimuth rotation is about a vertical axis, and elevation rotation is about an axis that rotates in the horizontal plane. The tracker is moved by two DC electric motors; each is controlled by a comparator circuit calculating the difference in resistance between a pair of partially shaded cadmium sulfide CdS photoresistors. One pair of photodiodes is responsible for the azimuth rotation, the other for the elevation rotation.

Before sunrise the Wattsun controller rotates the array in azimuth from where it stopped the previous

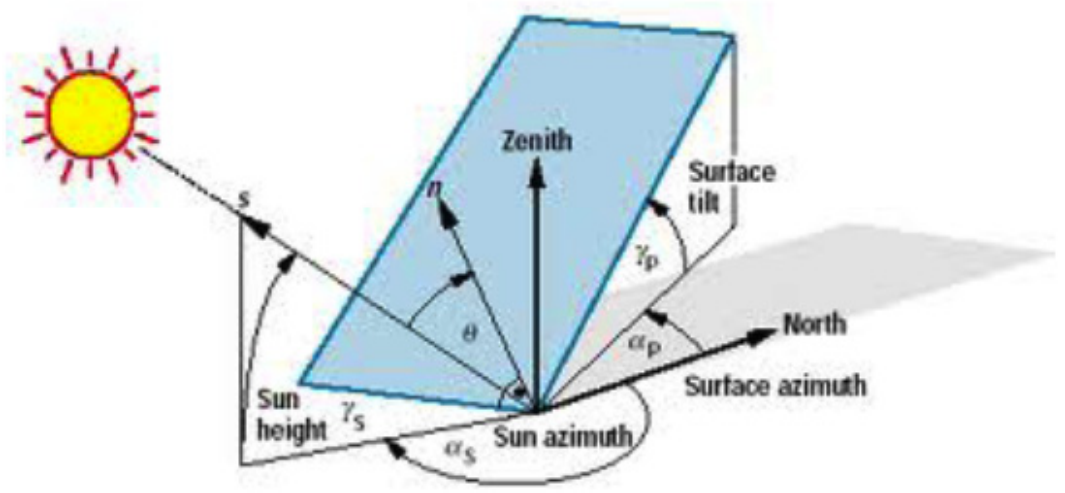

Figure 1: The angles to define the position of the sun and the orientation of the tilted plane [4]. 


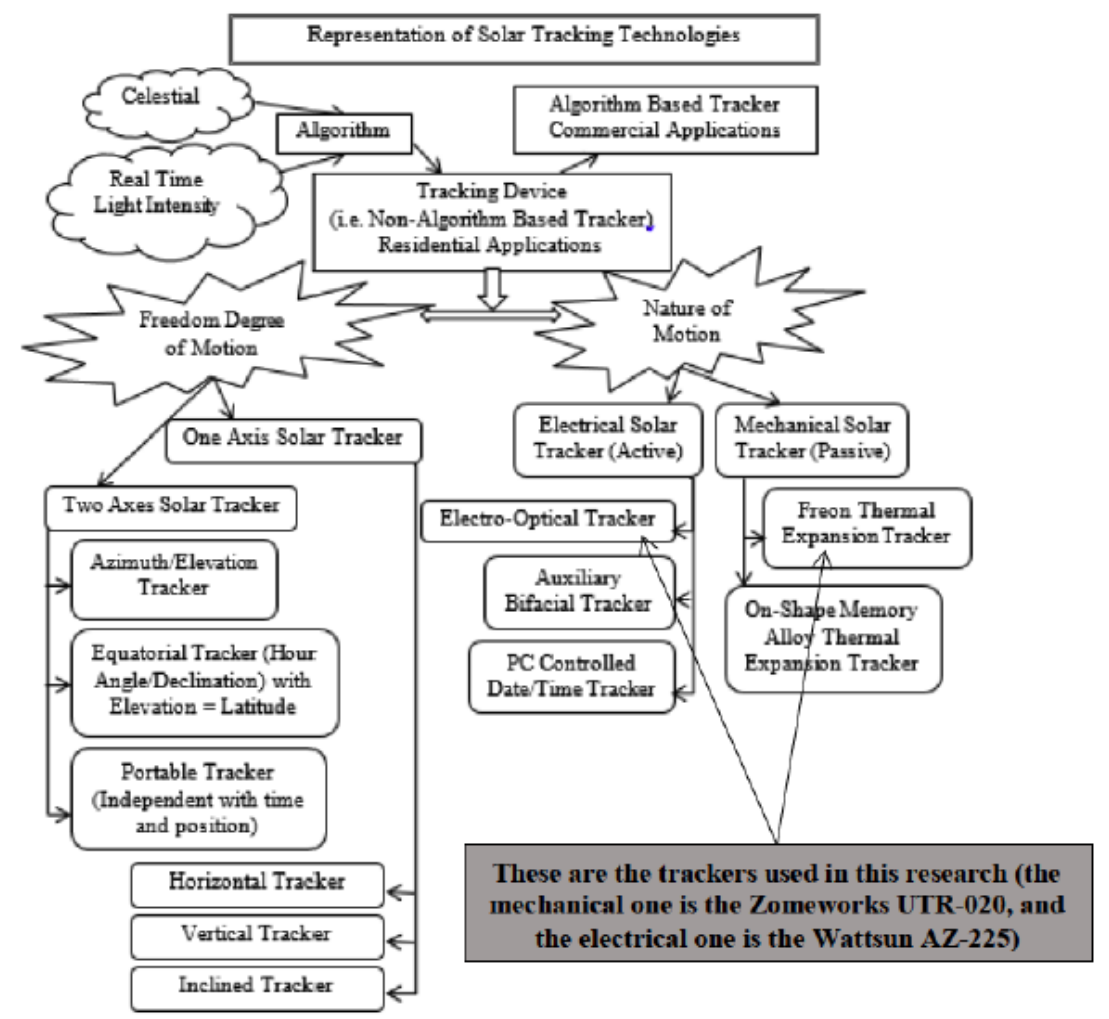

Figure 2: Representation of solar tracking technologies.

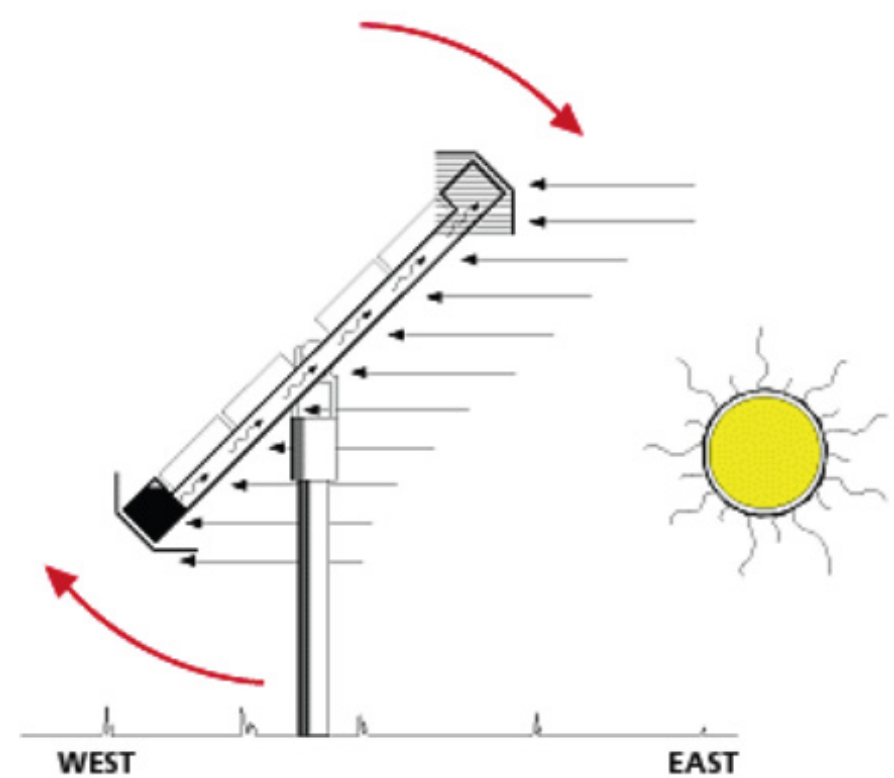

Figure 3: Image of the Zomeworks UTR-020 solar tracker showing the UTR facing west in the beginning of the day and the rising sun heats the un-shaded west-side canister, forcing liquid into the shaded east-side canister for the tracker to face east [5].

day to face east. As the morning sun's rays hit the two photodiode pairs, different light intensities produce resistance imbalances. The comparators sense these imbalances and the controller energizes the motors to balance these signals. This behavior of the Wattsun AZ-225 takes place throughout the day until sunset.

\subsection{Sun Angle Algorithms}

Sun angles were calculated from Michalsky's algorithm. (1988). This algorithm calculates true solar elevation and azimuth angles to a demonstrated accuracy of $0.01^{\circ}$ between 1950 and 2050. "The 
algorithm is taken from The Astronomical Almanac, which has published it as an addendum to their very accurate tabulations since 1984. It uses the same approach as an earlier paper by Walvern, but has a more simplified form" [6]. Walvern compared the declination angle in many commonly used algorithms as well. The declination angle is defined as "the angle between the line joining the centers of the sun and the earth and its projection on the equatorial plane" [6]. It was shown from this comparison that the Almanac Algorithm (AA) was the best of the simple algorithms that calculate solar position. "It is superior to the calculations based on least square fits to a given set of data since it is based on the long-term progression of the sun in the ecliptic plane" [6].

Reda and Andreas (2003) have developed a Solar Position Algorithm (SPA) to calculate the solar zenith and azimuth angles in the period from the year -2000 to 6000 , with uncertainties of $+/-0.0003^{\circ}$ [7]. This algorithm was not used in this study.

\subsection{Solar Tracking Error and Accuracy}

Many studies have used the power produced by a tracked PV array as a proxy to characterize tracker accuracy. Looking only at the electrical power, however, is not sufficient to determine tracking accuracy because the power output is a function of several variables, including irradiance, sky conditions, as well as tracker accuracy. Moreover, the lack of standards that describe tracker performance makes it difficult to evaluate and compare the performance of various solar trackers. Further, studies undertaken at different locations and times of the year have traditionally provided performance data under local conditions. "These challenges call for a method for accurately characterizing both absolute and relative tracking accuracy in the field, under a variety of weather conditions" [8].

Different tracking technologies likely have different accuracies. Algorithm based trackers, for example those that are used for pyrheliometers, are required to be of the highest accuracy. Non-algorithm based solar trackers utilize different sensing control schemes that introduce different tracking errors. Active vs. passive trackers likely vary in their ability to accurately follow the sun.

\section{RESEARCH METHODOLOGY}

Surface angles of two residential trackers were measured using potentiometers. Trackers specifications are summarized in Table 1 . The Zomeworks and Wattsun trackers held one and nine 224 W PV panels, respectively.

The measured angles were then compared to the sun angles calculated by Michalsky's celestial algorithm. Direct normal irradiance and global horizontal irradiance were also measured. Tracker accuracy was determined under a well-quantified solar resource.

The experiment was conducted at the Appalachian State University Solar Lab, Boone, NC between February 2013 and June 2013.

\subsection{Site Characteristics}

The Appalachian State University Solar Research Facility Laboratory in Boone, North Carolina (latitude $36.2167^{\circ} \mathrm{N}$, longitude $81.6747^{\circ} \mathrm{W}$, elevation $950 \mathrm{~m}$ ). The two trackers and a pyrheliometer are pole mounted and located within $15 \mathrm{~m}$ of each other as shown in Figure 4.

The site has a generally clear view of the southern sky. A row of trees blocked the sky from the $\mathrm{N}$ to the NW reducing diffuse radiation by around $20 \%$, as also seen in Figure 4.

The trackers and pyranometers receive some shading during the day. A site survey was conducted to identify sun angles at which shading was observed. Sun angles between $45^{\circ}$ and $225^{\circ}$ azimuth and below $36^{\circ}$ elevation, and sun azimuth angles greater than $270^{\circ}$, were excluded from the analysis.

\subsection{Solar Measurement Instrumentation}

Direct normal irradiance (DNI) was measured by a Hukesflux DR-1 pyrheliometer (first class) pointed at

Table 1: Specifications of the Solar Trackers Used in the Study

\begin{tabular}{|c|c|c|c|c|}
\hline Tracker & Az & El & Control & Drive \\
\hline \hline Zomeworks UTR-020 & Tracking & $40^{\circ}$ & Differential heating & Passive \\
\hline Wattsun AZ-225 & Tracking & Tracking & Differential irradiance & Active \\
\hline
\end{tabular}




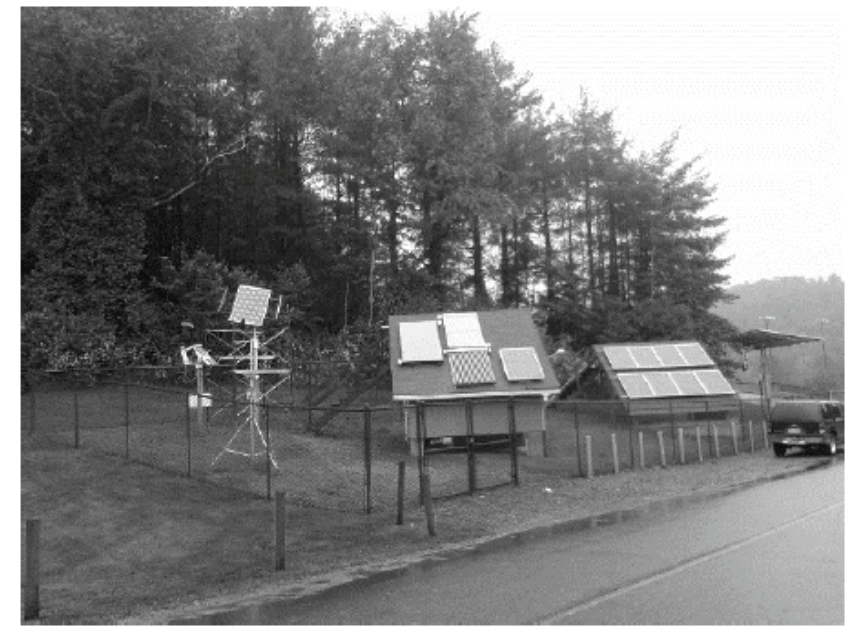

Figure 4: The ASU Solar Lab looking from the south showing the pyrheliometer (far left), one-axis tracker (left), and 2-axis tracker (far right). Trees to the north block around $20 \%$ of the sky.

the sun by a Minitrack II Solar Tracker. Positioning was by a microcontroller running Michalsky's celestial algorithm. Diffuse horizontal irradiance (DHI) was measured by a Hukseflux SR-11 pyranometer (first class).

\subsection{Angle Measurement Instrumentation}

The azimuth angle of the Zomeworks one-axis tracker was measured by a $150 \mathrm{~mm}, 10 \mathrm{k} \Omega$ linear potentiometer. The azimuth angle of the Wattsun twoaxis tracker was measured by capturing the rotation of the worm drive screw gear using a 10:1 gearbox and a 10 -turn, $100 \mathrm{k} \Omega$ rotating potentiometer. The elevation angle of the Wattsun two-axis tracker was measured by a $900 \mathrm{~mm}, 100 \mathrm{k} \Omega$ linear potentiometer. The potentiometers used to measure the azimuth angle of the Zomeworks tracker and the elevation angle of the Wattsun tracker converted the relative motion between the moving array and a fixed support into an electrical signal. The resistance of each potentiometer was measured using a $1 / 2$-bridge circuit implemented on a Campbell Scientific CR1000 data logger. Completion resistors were of the respective maximum potentiometer resistance. Excitation voltage was $2.5 \mathrm{~V}$.

\subsubsection{Calibration of the Potentiometers}

The objective of the calibration process was to get the resistance/angle transfer function of each of the potentiometers. Each tracker in turn was manually positioned at a known angle and its potentiometer output in the form of a ratio between the potentiometer voltage and the excitation voltage $\left(\mathrm{V}_{\text {pot }} / \mathrm{N}_{\mathrm{ex}}\right)$ was recorded. The transfer functions were empirically taken to be the quadratic best-fit curves.

\subsubsection{Calibration of the 1-Axis Tracker Azimuth} Potentiometer

A graph of one-axis tracker azimuth angle versus the potentiometer output is shown in Figure $\mathbf{5}$. The transfer function was given by:

$\theta_{1-\mathrm{Az}}\left({ }^{\circ}\right)=-1591.7\left(\frac{\mathrm{V}_{\text {pot }}}{\mathrm{V}_{\text {ex }}}\right)^{2}+1840.3\left(\frac{\mathrm{V}_{\text {pot }}}{\mathrm{V}_{\text {ex }}}\right)-296.85$

The main source of uncertainty in this equation was from determining the tracker angle, which was estimated to be $\pm 5^{\circ}$. The statistical uncertainty of the fit was $\pm 1^{\circ}$. Sensitivity was around $4.9 \mathrm{mV} /{ }^{\circ}$.

\subsubsection{Calibration of the 2-Axis Tracker Azimuth Potentiometer}

A graph of two-axis tracker azimuth angle versus the potentiometer output is shown in Figure 6 . The calibration equation was given by:

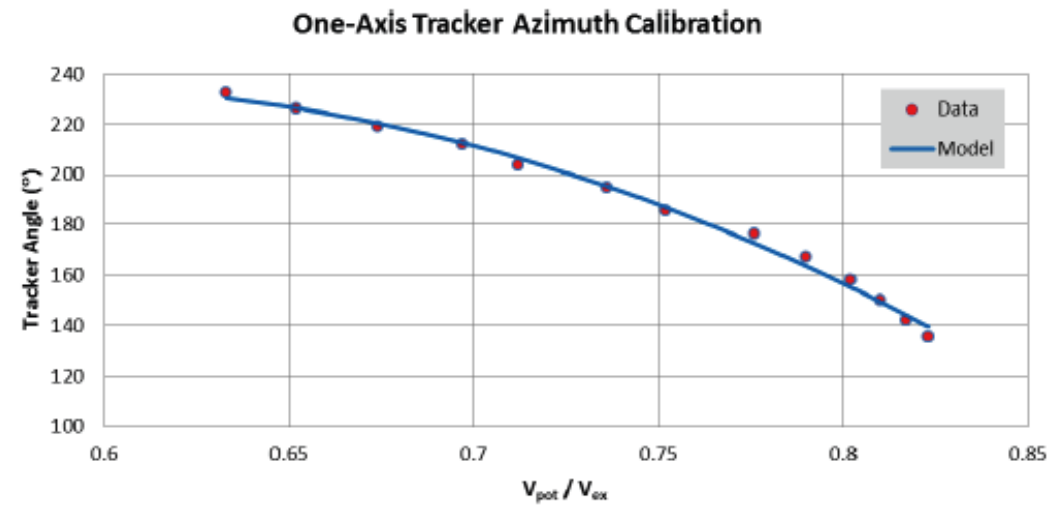

Figure 5: The relationship between the Zomeworks one-axis tracker azimuth angle and output of the $150 \mathrm{~mm}$ linear potentiometer showing 13 calibration points from $\left(0.825,140^{\circ}\right)$ to $\left(0.625,230^{\circ}\right)$. 
Two-Axis Tracker Azimuth Calibration

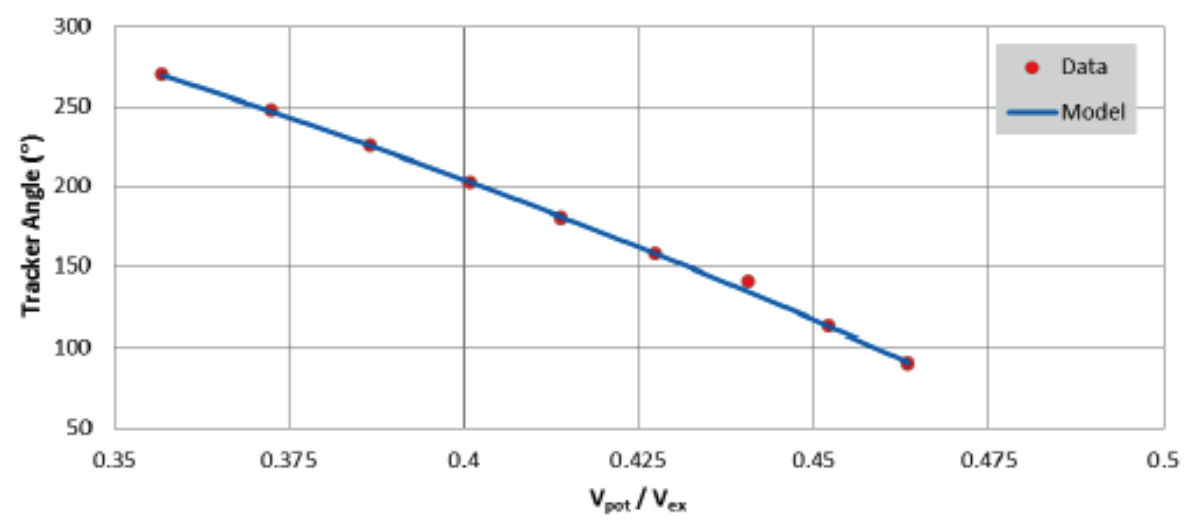

Figure 6: The relationship between Wattsun two-axis tracker azimuth angle and output of the rotating potentiometer showing 9 calibration points from $\left(0.475,70^{\circ}\right)$ to $\left(0.35,280^{\circ}\right)$.

$\theta_{2-\mathrm{Az}}\left({ }^{\circ}\right)=-2409.5\left(\frac{\mathrm{V}_{\mathrm{pot}}}{\mathrm{V}_{\mathrm{ex}}}\right)^{2}+297.19\left(\frac{\mathrm{V}_{\mathrm{pot}}}{\mathrm{V}_{\mathrm{ex}}}\right)+470.69$

The main source of uncertainty in this equation was from determining the tracker angle, which was estimated to be $\pm 3^{\circ}$. The statistical uncertainty of the fit was $\pm 1^{\circ}$. Sensitivity was around $1.5 \mathrm{mV} /{ }^{\circ}$.

\subsubsection{Calibration of the 2-Axis Tracker Elevation} Potentiometer

A graph of two-axis tracker elevation angle versus the potentiometer output is shown in Figure 7. The calibration equation was given by:

$\theta_{2-\mathrm{El}}\left({ }^{\circ}\right)=281.53\left(\frac{\mathrm{V}_{\text {pot }}}{\mathrm{V}_{\text {ex }}}\right)^{2}+8.4164\left(\frac{\mathrm{V}_{\text {pot }}}{\mathrm{V}_{\text {ex }}}\right)+6.3035$

The main source of uncertainty in this equation was from determining the tracker angle, which was estimated to be $\pm 3^{\circ}$. The statistical uncertainty of the fit was $\pm 1^{\circ}$. Sensitivity was around $14.4 \mathrm{mV} /{ }^{\circ}$.

\subsection{Data Collection}

\subsubsection{Sample}

The data sample included one minute averages calculated from a 10 second sampling rate. Measured values include the three tracker angles, DNI, and DHI. In addition, for each minute the sun azimuth and elevation angles were calculated using Michalsky's celestial algorithm. The data collection period was from February $10^{\text {th }}$ to June $16^{\text {th }}, 2013$ for the azimuth angle of the Zomeworks tracker (195,272 minutes), and from May $16^{\text {th }}$ to June $16^{\text {th }}, 2013$ for Wattsun tracker $(46,081$ minutes).

\subsubsection{Data Processing}

The data processing process consisted of validating and filtering. The input was one-minute data in text

Two-Axis Tracker Elevation Calibration

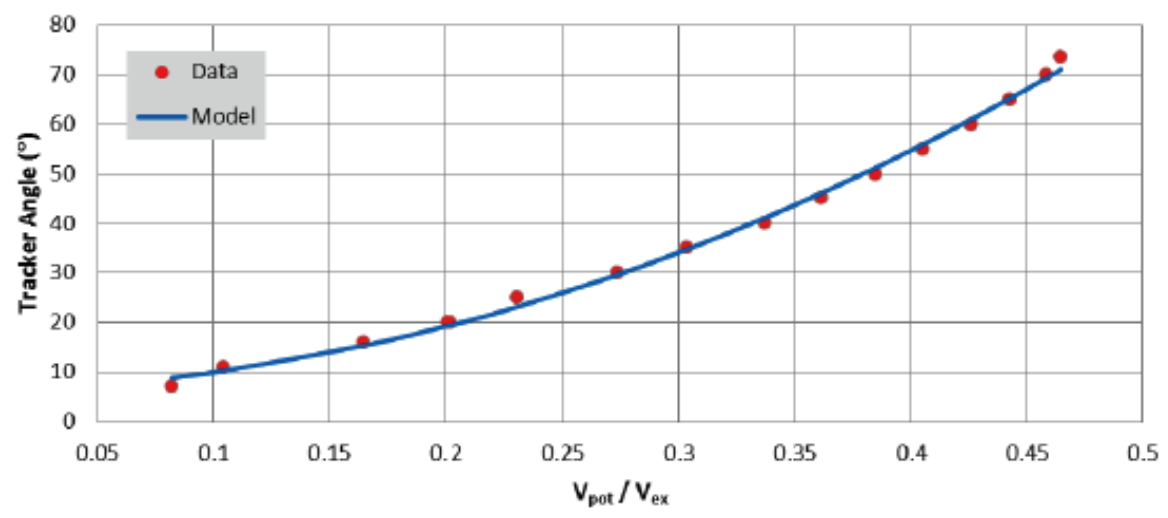

Figure 7: The relationship between Wattsun two-axis tracker elevation angle and output of the $900 \mathrm{~mm}$ linear potentiometer showing 15 calibration points from $\left(0.075,9^{\circ}\right)$ to $\left(0.45,68^{\circ}\right)$. 
format generated by the data acquisition system. The validation process identified 1-minute intervals for which the sun angles were between sunrise and sunset and the DBF, defined as

$\mathrm{DBF}=\frac{D N I_{\perp}}{\mathrm{GHI}}$

was between 0 and $1 . \mathrm{GHI}$ is the Global Horizontal Irradiance, and $\mathrm{DNI}_{\perp}$ is the component of DNI normal to the horizontal. This process eliminated non-physical values. A filtering process was then applied to reduce statistical spread to better show correlations. Oneminute intervals were required to have a $\mathrm{GHI}$ greater than $100 \mathrm{~W} / \mathrm{m}^{2}$. In addition, 1-minute intervals that met the shading condition were filtered out. After validation and filtering processes were executed, the remaining data sets included 32,405 minutes ( $17 \%$ of total) for the Zomeworks tracker and 14,118 minutes ( $31 \%$ of total) for the Wattsun tracker.

\subsection{Data Analysis Procedures}

The schematic in Figure 8 summarizes the data analysis methodology.

This study was guided by one primary research question (RQ). Two related research hypotheses $(H)$ were generated for the study:

$R Q$ : What is the accuracy of non-algorithm based one-axis and two-axis solar trackers under varying DBF and $\mathrm{GHI}$ ?

H1. There will be strong positive correlations between tracker accuracy and level of DBF for the
Wattsun AZ-225 active altitude and azimuth and the Zomeworks UTR-020 passive azimuth solar trackers.

H2. The Wattsun AZ-225 active altitude and azimuth tracker will be more accurate than the Zomeworks UTR-020 passive azimuth tracker under strong DBF and $\mathrm{GHI}$.

The DBF, tracking error percentage $\mathrm{E}$, and angle accuracy percentage $A$ were calculated on a oneminute basis according to:

$\mathrm{DBF}=\frac{\mathrm{DNI} \sin \gamma_{\mathrm{s}}}{\mathrm{DNI} \sin \gamma_{\mathrm{s}}+\mathrm{GHI}}$,

where $\gamma_{s}$ is the solar elevation angle,

$E=\frac{\left(\theta_{\text {meas }}-\theta_{\text {calc }}\right)}{\theta_{\text {calc }}} \times 100 \%$,

where $\theta_{\text {meas }}$ is the angle measured by the potentiometer and $\theta_{\text {calc }}$ is the angle calculated by Michalsky's celestial algorithm, and

$A=100 \%-|E|$

Correlations $r$ are calculated from

$r=\frac{1}{n-1} \sum\left(\frac{x_{i}-\bar{x}}{s_{x}}\right)\left(\frac{y_{i}-\bar{y}}{s_{y}}\right)$

for a sample size $n$, where $x_{i}$ and $y_{i}$ are the targeted variables, $\bar{x}$ and $\bar{y}$ are the sample means of the targeted variables, and $s_{x}$ and $s_{y}$ are the standard deviations of the targeted variables.

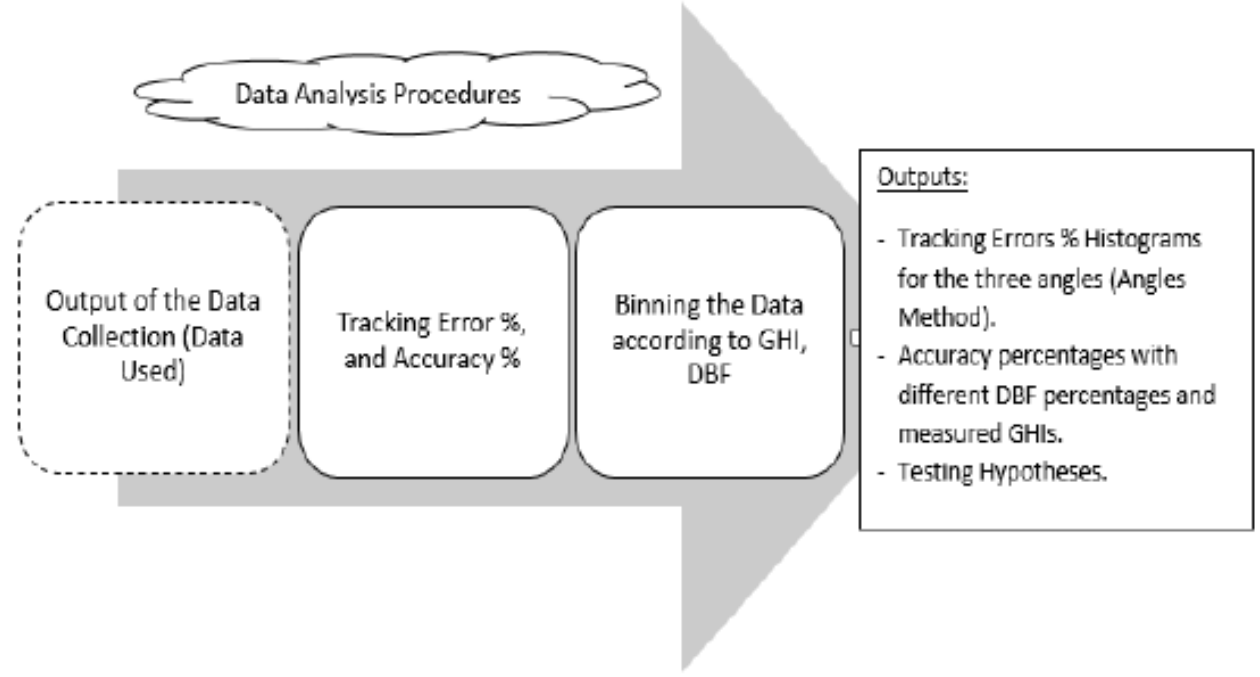

Figure 8: A diagram of the data analysis procedures. 


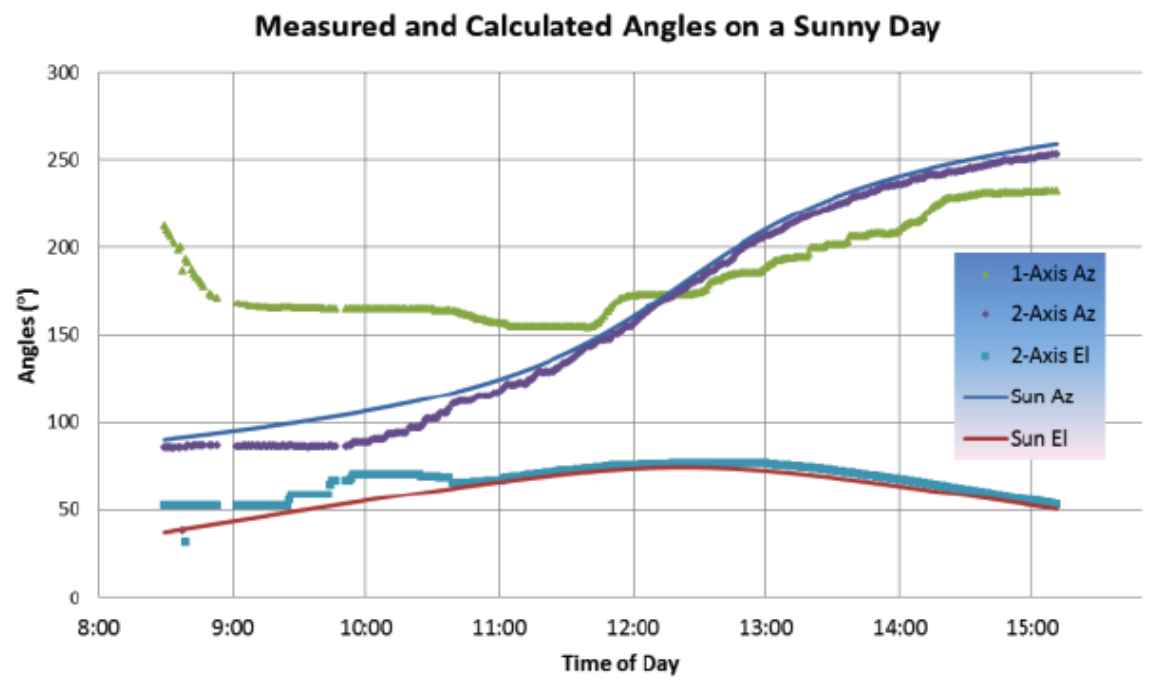

Figure 9: Measured tracker and calculated sun angles on a sunny day (May 24, 2013).

Average tracker error and accuracy were calculated based on bins of DBF and GHI. The reason for having these different categories was to investigate tracker performance in different weather conditions.

\section{RESEARCH FINDINGS}

\subsection{Time-Ordered Tracker Behavior}

Figure 9 shows the behavior of the measured tracker angles overlaid with calculated sun angles from Michalsky on a representative sunny day, May 24, 2013. It is assumed that on this day DNI was sufficient to result in the maximum tracker accuracy.

Angular deviations are shown in Figure 10. The twoaxis azimuth and elevation angles were generally within $5^{\circ}$ of the calculated sun angle (read on the right axis). The two-axis tracker tended to slightly lag the sun in azimuth, and overshooting in elevation. The greatest overshot was seen in the morning.

It is clear from Figure $\mathbf{1 0}$ that the one-axis tracker has significantly greater error - as much as $60^{\circ}$ (read on the left axis). The tracker led the sun in azimuth (pointing too far west) throughout the morning with decreasing deviation, had the smallest deviations around noontime, and lagged the sun in azimuth (pointing too far east) throughout the afternoon with increasing deviation.

One likely physical explanation of the large morning error is that in the morning the tracker is still looking to the west where it was positioned as the sun set on the

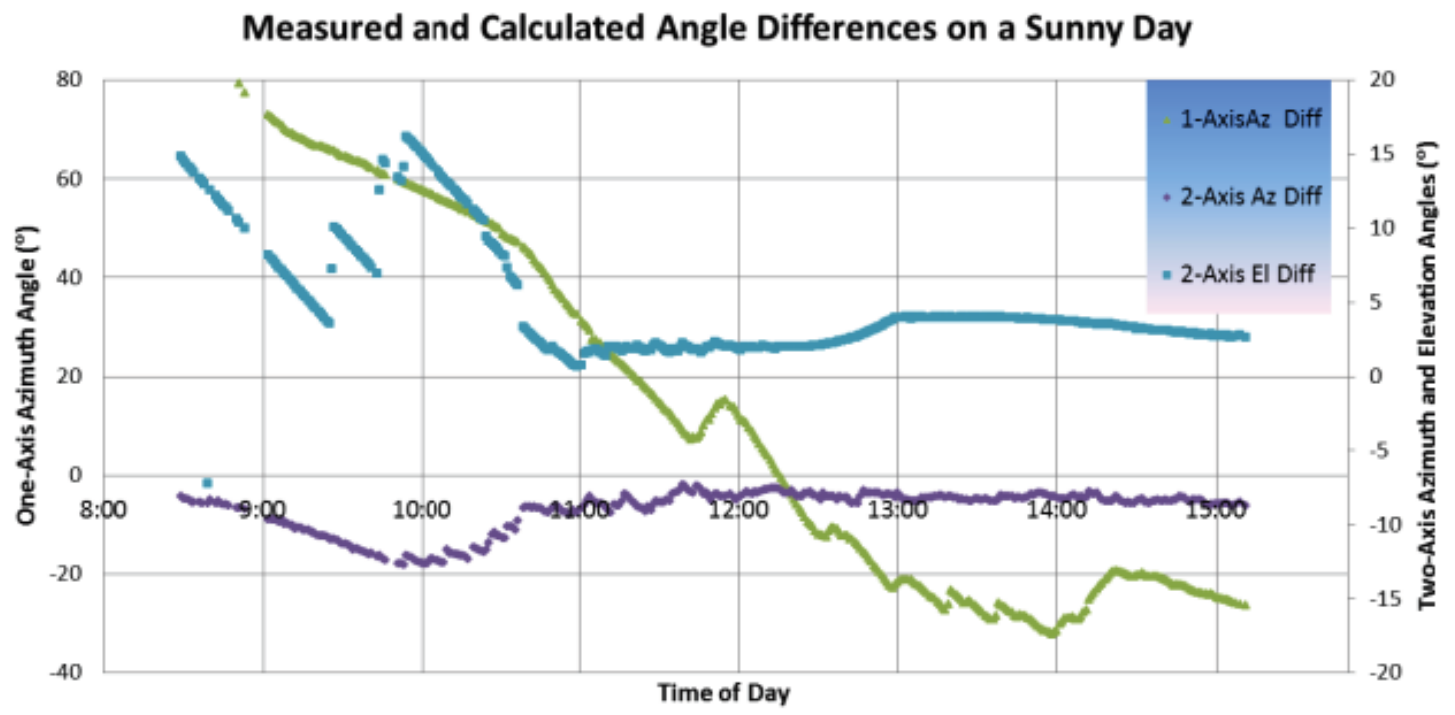

Figure 10: Angular deviations on a sunny day (May 24, 2013). 
Tracking Error Percentage Histogram of Zomeworks UTR-020 Azimuth

Angle

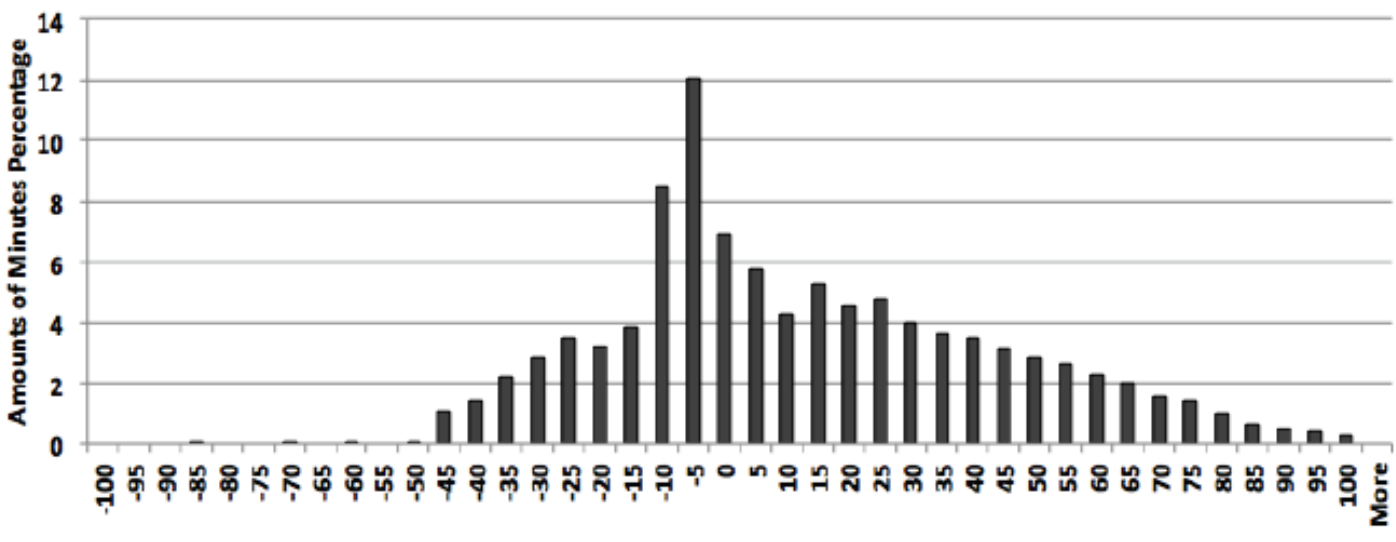

Tracking Error \%

Figure 11: Tracking error percentage histogram of Zomeworks UTR-020 azimuth angle showing different behaviors throughout the day with errors from $-10 \%$ to $+10 \%$ during $33.1 \%$ of the data collection time period.

previous day. Consequently, the need for the greatest rotation occurs when irradiance is low and the driving torque is weak.

\subsection{Tracking Error Histograms}

\subsubsection{One Axis Zomeworks UTR-020 Azimuth Angles}

Figure 11 shows a histogram of the azimuth tracking errors of the Zomeworks one-axis tracker. The histogram is centered near zero error and approximately follows a normal distribution with a large standard deviation. The distribution is skewed toward positive tracker error. The average tracking error is $+25 \%$, the median tracking error is $+19 \%$, and the dominant mode is $-5 \%$ tracking error.
To investigate the morning lead and afternoon lag behavior seen in Figure 10, hourly histograms of tracking error were produced and overlaid, as shown in Figure 12. Tracking error clearly starts the day with large positive values (tracker pointing west of the sun angle), and the error systematically decreases throughout the morning. The tracking error is near zero at noontime. After noon the tracking error is negative (tracker pointing east of the sun angle) and increases in magnitude.

\subsubsection{Two Axis Wattsun AZ-225 Azimuth Angle}

Figure 13 shows a histogram of the azimuth tracking errors of the Wattsun solar tracker. The histogram is centered near zero and approximately follows a normal distribution with a relatively small

\section{Daily Evolution of Zomeworks Azimuth Error}

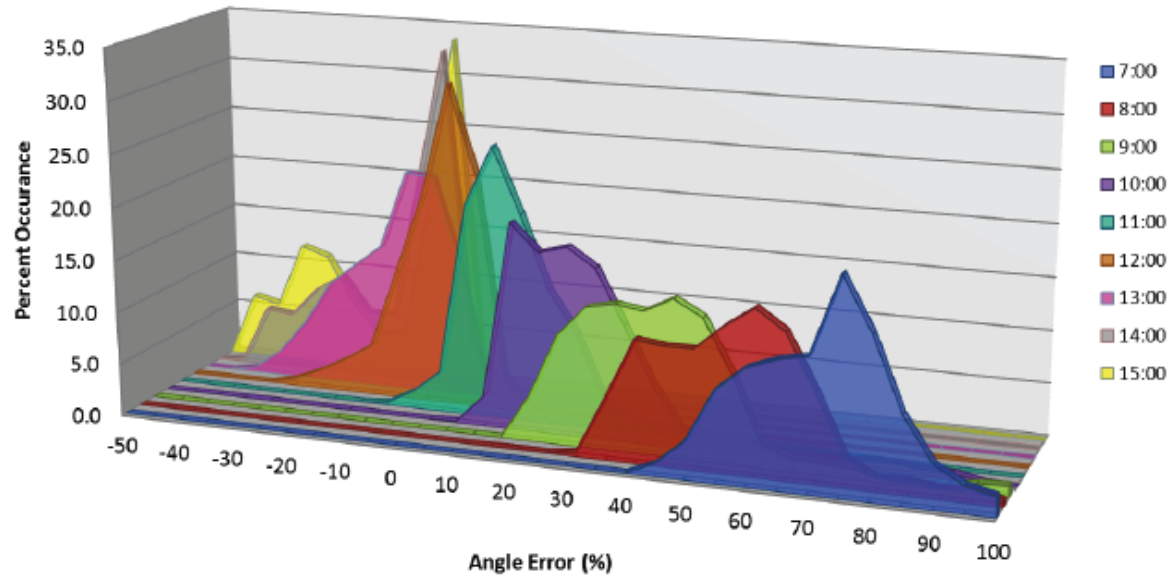

Figure 12: Tracking error percentage histogram of Zomeworks UTR-020 azimuth angle per hour shows that the tracker leads the sun in the morning and lags the sun in the afternoon. 


\section{Tracking Error Percentage Histogram of Wattsun AZ-225 Azimuth Angle}

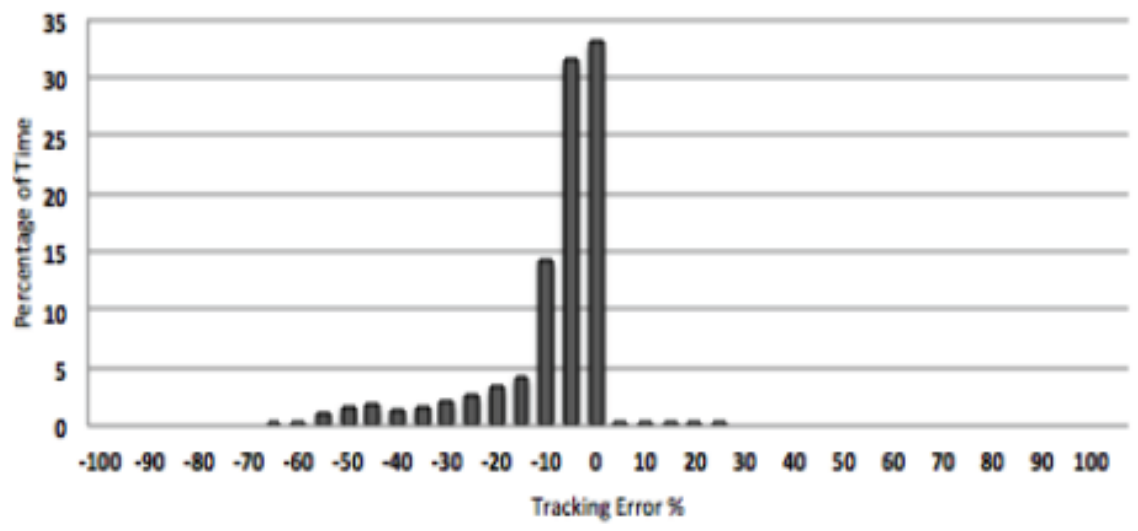

Figure 13: Tracking error percentage histogram of Wattsun AZ-225 azimuth angle showing errors from $-10 \%$ to $+10 \%$ during $79.3 \%$ of the data collection time period.

standard deviation. The distribution is skewed toward negative tracker error. The average tracking error is $12 \%$, the median tracking error is $-7 \%$, and the dominant mode is $0 \%$ tracking error. Azimuth tracking rarely leads the sun azimuth angle, but lags in azimuth angle (too far east) by more than $10 \%$ around $15 \%$ of the time.

\subsubsection{Two Axis Wattsun AZ-225 Elevation Angle}

Figure 14 shows a histogram of the elevation tracking errors of the Wattsun solar tracker. The histogram is generally normally distributed with a standard deviation larger than in azimuth, and skewed toward negative tracker error. The average tracking error of $+11 \%$, the median tracking error is $+6 \%$, and the dominant mode is $+5 \%$ tracking error. Elevation tracking seems to over- and under-shoot the sun position by greater than $10 \%$ with approximately equal frequency - less than $10 \%$ of the time in each case. In addition, gross overshooting is occasionally experienced.

\section{CONCLUSIONS}

In order to answer the research questions, tracker accuracy was binned in both Global Horizontal Irradiance $(\mathrm{GHI})$ and Direct Beam Fraction (DBF). Weighted average accuracies were calculated for each bin. Tables 2, 3, and 4 show the results for the three measured angles.

Table 2 shows that for the Zomeworks tracker the accuracy increases as $\mathrm{GHI}$ increases for all values of DBF. This suggests that working fluid heating is directly proportional to irradiance. In addition, accuracy decreases as DBF increases for low GHI. This inverse

\section{Tracking Error Percentage Histogram of Wattsun AZ-225 Elevation Angle}

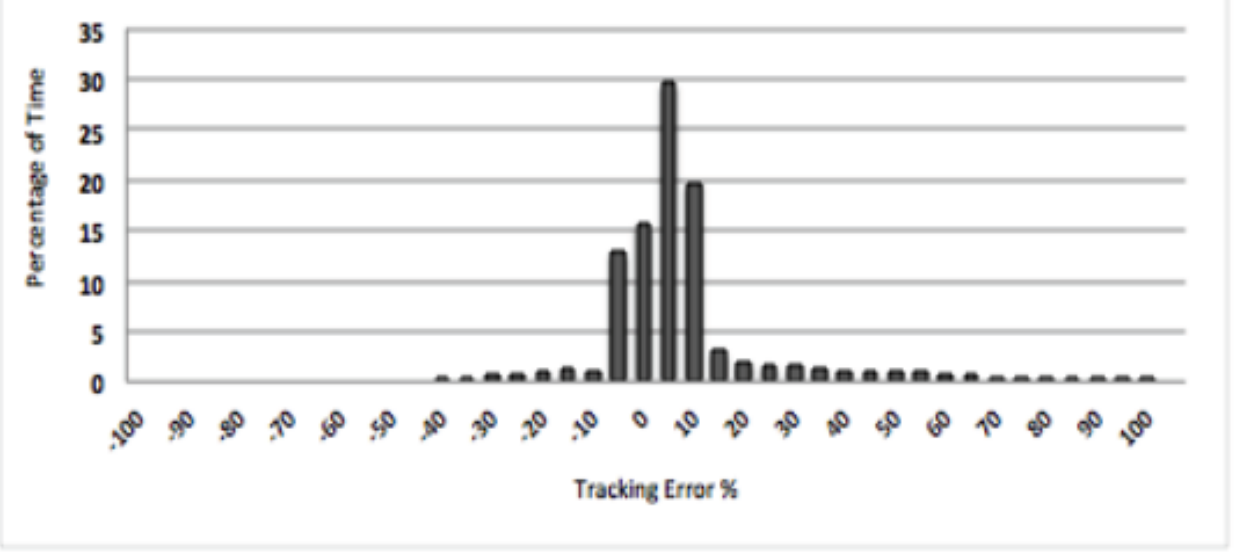

Figure 14: Tracking error percentage histogram of Wattsun AZ-225 elevation angle showing errors from $-10 \%$ to $+10 \%$ during $59.2 \%$ of the data collection time period. 
Table 2: Zomeworks UTR-020 Percent Accuracy for Varying DBF and GHI (Typical Statistical Uncertainty = 3\%)

\begin{tabular}{|c|c|c|c|c|c|c|c|}
\hline \multirow[t]{2}{*}{ DBF (\%) } & \multicolumn{6}{|c|}{$\mathrm{GHI}\left(\mathrm{W} / \mathrm{m}^{2}\right)$} & \multirow{2}{*}{$\begin{array}{l}\text { Weighted } \\
\text { Average }\end{array}$} \\
\hline & $100-300$ & $300-500$ & $500-700$ & $700-800$ & $900-1100$ & $1100-1300$ & \\
\hline 010 & 68 & 77 & 82 & $\mathrm{~N}$ & $\mathrm{~N}$ & $\mathrm{~N}$ & 70 \\
\hline $20-30$ & 51 & 75 & 81 & 81 & $\mathrm{~N}$ & $\mathrm{~N}$ & 76 \\
\hline $30-40$ & 44 & 68 & 81 & 84 & $\mathrm{~N}$ & $\mathrm{~N}$ & 73 \\
\hline $50-60$ & 46 & 56 & 73 & 85 & 84 & 85 & 77 \\
\hline $60-70$ & 27 & 46 & 63 & 83 & 87 & 90 & 74 \\
\hline $70-80$ & 33 & 41 & 62 & 79 & 88 & 92 & 70 \\
\hline $80-90$ & $\mathrm{~N}$ & 44 & 65 & 85 & 91 & 93 & 83 \\
\hline Weighted Average & 67 & 65 & 70 & 83 & 90 & 90 & 75 \\
\hline
\end{tabular}

$\mathrm{N}$ : No data in the category.

Table 3: Wattsun AZ-225 Azimuth Angle Percent Accuracy for Varying DBF and GHI (Typical Statistical Uncertainty = $3.5 \%)$

\begin{tabular}{|c|c|c|c|c|c|c|c|}
\hline \multirow[t]{2}{*}{ DBF (\%) } & \multicolumn{6}{|c|}{$\mathrm{GHI}\left(\mathrm{W} / \mathrm{m}^{2}\right)$} & \multirow{2}{*}{$\begin{array}{l}\text { Weighted } \\
\text { Average }\end{array}$} \\
\hline & $100-300$ & $300-500$ & $500-700$ & $700-800$ & $900-1100$ & $1100-1300$ & \\
\hline 010 & 76 & 82 & 83 & $\mathrm{~N}$ & $\mathrm{~N}$ & $\mathrm{~N}$ & 78 \\
\hline $20-30$ & 94 & 93 & 92 & 91 & 91 & $\mathrm{~N}$ & 92 \\
\hline $30-40$ & 94 & 94 & 94 & 94 & 87 & $\mathrm{~N}$ & 94 \\
\hline $50-60$ & 90 & 92 & 93 & 94 & 93 & 91 & 93 \\
\hline $60-70$ & 96 & 93 & 94 & 94 & 94 & 94 & 94 \\
\hline $70-80$ & 94 & 95 & 93 & 95 & 95 & 95 & 94 \\
\hline $80-90$ & 96 & 94 & 92 & 95 & 95 & 95 & 95 \\
\hline $90-100$ & $\mathrm{~N}$ & $\mathrm{~N}$ & 96 & 98 & 98 & $\mathrm{~N}$ & 98 \\
\hline
\end{tabular}

$\mathrm{N}$ : No data in the category.

Table 4: Wattsun AZ-225 Elevation Angle Percent Accuracy for Varying DBF and GHI (Typical Statistical Uncertainty = $1.4 \%)$

\begin{tabular}{|c|c|c|c|c|c|c|c|}
\hline \multirow[t]{2}{*}{ DBF (\%) } & \multicolumn{6}{|c|}{$\mathrm{GHI}\left(\mathrm{W} / \mathrm{m}^{2}\right)$} & \multirow{2}{*}{$\begin{array}{c}\text { Weighted } \\
\text { Average }\end{array}$} \\
\hline & $100-300$ & $300-500$ & $500-700$ & $700-800$ & $900-1100$ & $1100-1300$ & \\
\hline 010 & 75 & 89 & 95 & $\mathrm{~N}$ & $\mathrm{~N}$ & $\mathrm{~N}$ & 79 \\
\hline $20-30$ & 89 & 93 & 95 & 95 & 99 & $\mathrm{~N}$ & 93 \\
\hline $30-40$ & 92 & 94 & 96 & 95 & 97 & $\mathrm{~N}$ & 95 \\
\hline $50-60$ & 91 & 94 & 95 & 96 & 96 & 96 & 95 \\
\hline $60-70$ & 95 & 94 & 94 & 97 & 96 & 96 & 96 \\
\hline $70-80$ & 94 & 92 & 93 & 97 & 96 & 96 & 95 \\
\hline $80-90$ & 99 & 92 & 93 & 96 & 96 & 97 & 96 \\
\hline $90-100$ & $\mathrm{~N}$ & $\mathrm{~N}$ & 94 & 95 & 95 & 95 & 95 \\
\hline
\end{tabular}

$\mathrm{N}$ : No data in the category. 
Table 5: First Hypothesis Test Results

\begin{tabular}{|c|c|c|c|}
\hline Angle Measured & Correlation Coefficient Between Accuracy and DBF & Correlation & Result \\
\hline \hline Zomeworks Azimuth & +0.2 & Weak Positive & Rejected \\
\hline Wattsun Azimuth & +0.6 & Strong Positive & Accepted \\
\hline Wattsun Elevation & +0.4 & Moderate Positive & Rejected \\
\hline \multicolumn{2}{|c|}{ Final Result } & Hypothesis Rejected \\
\hline
\end{tabular}

relationship between accuracy and DBF of the Zomeworks tracker may be explained by the shape of the aluminum shadow plates that surround the working fluid canisters. As these shadow plates are not parabolic they do not necessarily focus direct radiation, and may more effectively reflect diffuse radiation to the canisters. The overall weighted average of the accuracy of the Zomeworks UTR-020 azimuth angle is $75 \%$.

Both Tables 3 and $\mathbf{4}$ show that for the Wattsun tracker the weighted averages of the azimuth and elevation accuracies increase slightly with increasing GHI. In addition, the weighted averages of the accuracies increase significantly as DBF increased up to $30 \%$, and are consistently high for DBF greater than $30 \%$. The overall weighted averages of the accuracies of the Wattsun AZ-225 azimuth and elevation angles were $88 \%$ and $89 \%$ respectively.

\subsection{Testing Hypothesis}

Table 5 shows that the correlation coefficients between the Zomeworks azimuth, Wattsun azimuth, and Wattsun elevation angle accuracies and DBF are +0.2 , +0.6, and +0.4, respectively. Because the correlation coefficient of +0.6 is the only coefficient that describes strong correlation, the first hypothesis is rejected.

In order to test the second hypothesis, the weighted average accuracies of the Zomeworks and Wattsun azimuth tracking were plotted against DBF (Figure 15) and $\mathrm{GHI}$ (Figure 16) under all conditions, the Wattsun tracker is more accurate in azimuth compared to the Zomeworks tracker. Therefore, the second hypothesis is accepted.

\section{FUTURE RESEARCH}

This research was the first of its kind to fill a gap in the literature by determining the accuracy of solar trackers through direct measurement of their tracking angles. Because this research was done only on two types of solar trackers, further studies could be done on other types of solar trackers. Additional studies could compare the accuracy of solar trackers determined by the "Angles Method" as this research did

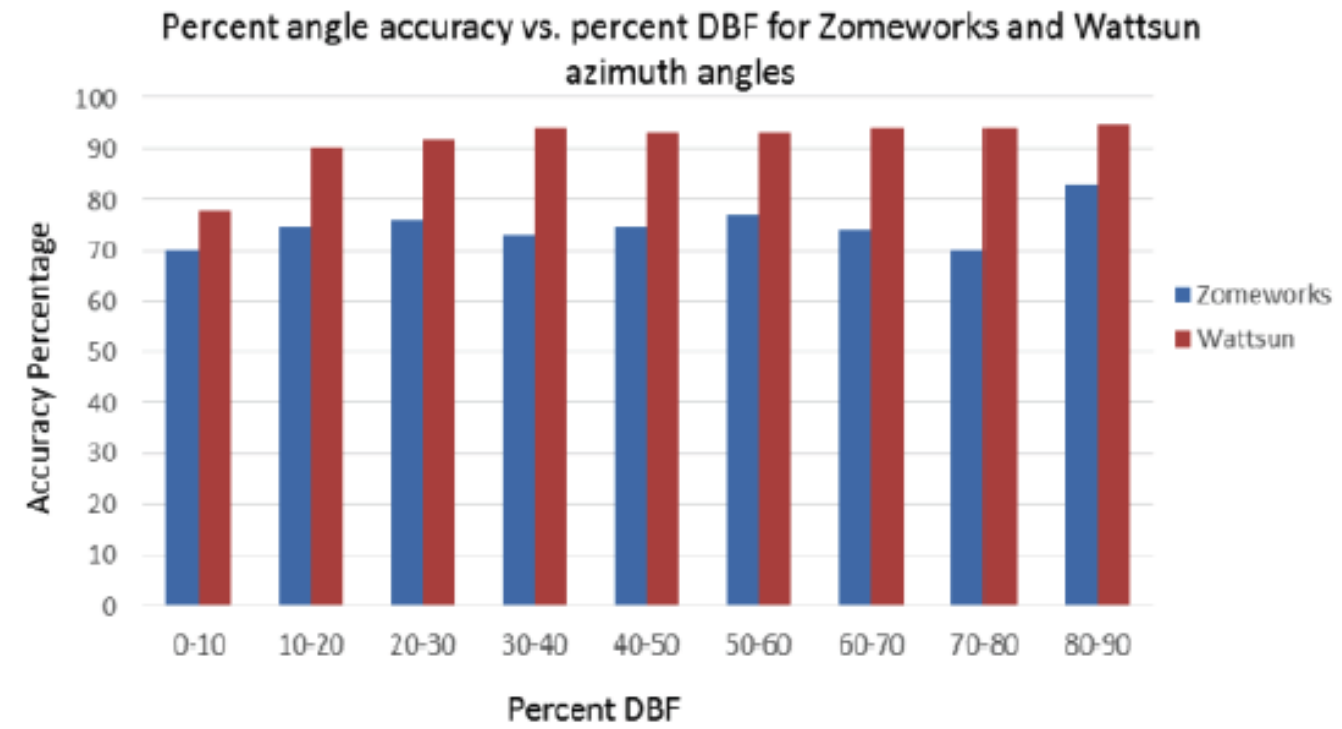

Figure 15: Percent angle accuracy vs. percent DBF for Zomeworks and Wattsun azimuth angles. 


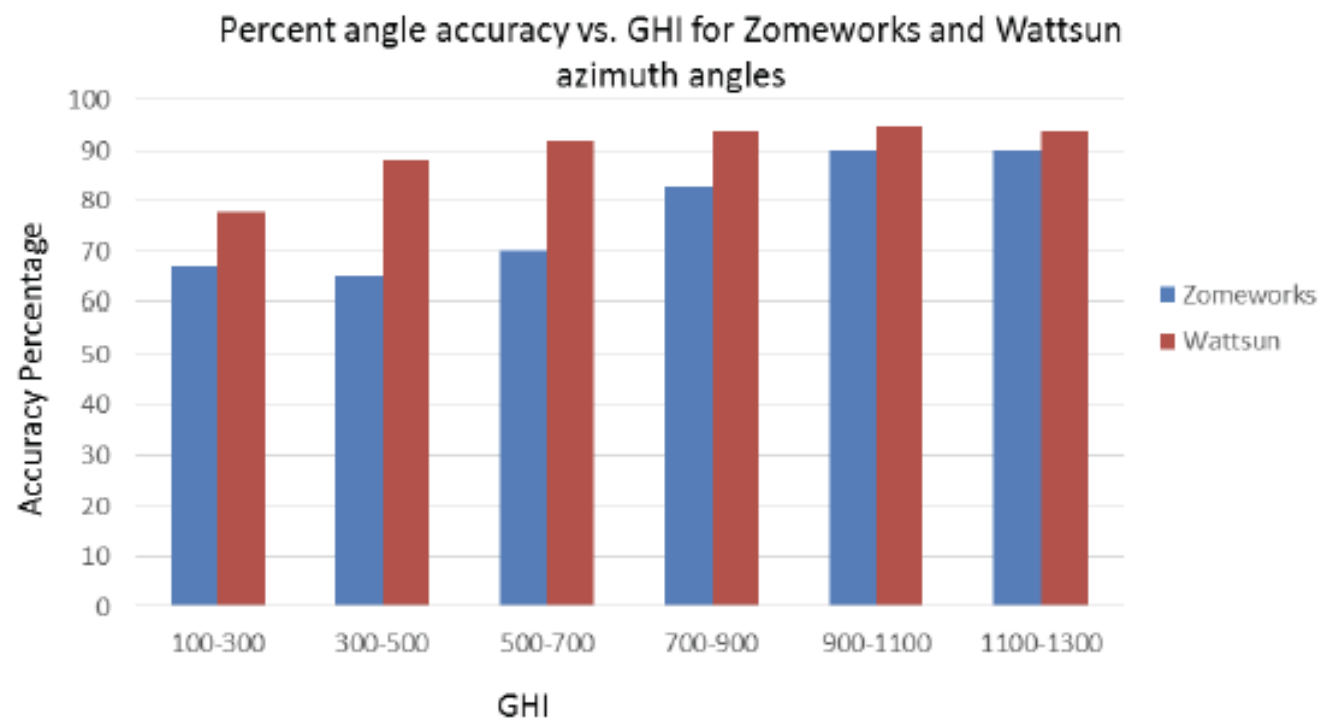

Figure 16: Percent angle accuracy vs. GHI for Zomeworks and Wattsun azimuth angles.

and the "Maximum Power Point (MPP) Method" as previous studies did. In addition, this research could be repeated taking into consideration other variables like wind speed, and wind direction.

Another focus of future research could be to examine the economic values of solar trackers under various climatic conditions by extrapolating the results of this study. Customer knowledge of solar tracker's accuracy could improve the solar PV market.

Several design modifications of the Zomeworks UTR-020 could be tested as well. The cross section of the canisters could be changed to explore better heating of the working fluid. Different selective absorptive coatings of these canisters could be tested for improved heating of the working fluid. Different shapes, materials, or coatings of the aluminum shadow plates could be tested for enhanced reflectivity. New materials could be tested for the working fluids. Further research could be done to investigate different nanomaterials for use as the Zomeworks working fluid.

\section{ACKNOWLEDGEMENT}

The authors would like to thank the Appalachian State University Appalachian Energy Center and the Department of Technology and Environmental Design for supporting this work.

\section{APPENDIX}

$$
\begin{array}{ll}
\alpha_{p} & =\text { Surface Azimuth Angle } \\
\gamma_{p} & =\text { Surface Elevation Angle }
\end{array}
$$

$$
\begin{aligned}
& \alpha_{\mathrm{s}} \quad=\quad \text { Solar Azimuth Angle } \\
& \gamma_{\mathrm{s}}=\text { Solar Elevation Angle } \\
& \theta=\text { Incident Angle } \\
& \mathrm{V}_{\text {pot }}=\text { Potentiometer Voltage } \\
& \mathrm{V}_{\mathrm{ex}} \quad=\text { Excitation Voltage } \\
& \theta_{1-A z}=\text { Azimuth Angle of One Axis Solar Tracker } \\
& \theta_{2-A z}=\text { Azimuth Angle of Two Axis Solar Tracker } \\
& \theta_{2-\mathrm{El}}=\text { Elevation Angle of Two Axis Solar Tracker } \\
& D N I_{\perp} \quad=\quad \text { Direct Normal Irradiance perpendicular to } \\
& \text { the surface } \\
& E=\text { Tracking Error Percentage (-/+) } \\
& \theta_{\text {meas }}=\text { Angle measured by the potentiometer } \\
& \theta_{\text {calc }}=\text { Angle calculated by Michalsky's Algorithm } \\
& \text { A }=\text { Angle Accuracy Percentage } \\
& |E| \quad=\text { Absolute Value of } E \\
& r \quad=\text { Correlation Coefficient } \\
& n=\text { Data sample size } \\
& \mathrm{x}_{\mathrm{i}}=\text { First Targeted Variable } \\
& \mathrm{y}_{\mathrm{i}} \quad=\quad \text { Second Targeted Variable } \\
& \bar{x} \quad=\quad \text { Mean of First Targeted Variable }
\end{aligned}
$$




\section{$\bar{y} \quad=\quad$ Mean of Second Targeted Variable \\ $\mathrm{s}_{\mathrm{x}} \quad=$ Standard Deviation of First Targeted Variable \\ $\mathrm{S}_{\mathrm{y}} \quad=$ Standard Deviation of Second Targeted Variable}

\section{REFERENCES}

[1] Mousazadeh $H$, Keyhani A, Javadi A, Molli H, Abriana, K, Sharifi A. A review of principle and sun-tracking methods for maximizing solar systems output. Renewable and Sustainable Energy Reviews 2009; 13(8): 1800-18. http://dx.doi.org/10.1016/j.rser.2009.01.022

[2] Clifford MJ, Eastwood D. Design of a novel passive solar tracker. Solar Energy 2004; 77(3): 269-80.

http://dx.doi.org/10.1016/j.solener.2004.06.009

[3] Robinson J, Raichle B. Performance comparison of fixed, 1-, and 2- axis tracking systems for small photovoltaic systems with measured direct beam fraction. In: Appalachian State University. Proceedings of the World Renewable Energy Forum, WREF2012, 13-17 May 2012, Denver, CO, USA. Boulder, Colorado; 2012. Available from http://tec.appstate.edu/sites/tec.appstate.edu/files/pv\%20trac king $\% 20$ system $\% 20$ performance $\% 20$ and $\% 20$ direct $\% 20$ bea m\%20robinson\%20raichle.pdf
[4] Renewable Energy World. Technology Fundamentals: The Sun as an Energy Resource: The angles to define the position of the sun and the orientation of the tilted plane [image]. http://www.volker-quaschning.de/articles/ fundamentals1/index_e.php (accessed May 2003).

[5] GoGreenSolar [homepage on the internet]. Solar Tracker: Zomeworks Track Rack UTR020 Universal Solar Tracker, 20 Sq. Ft. [image]. Placentia, CA, USA: Gigawatt, Inc.; c20062014: Available from http://www.gogreensolar.com/ products/zomeworks-track-rack-utr020-universal-solartracker-20-sq-ft

[6] Michalsky J. The astronomical almanac's algorithm for approximate solar position (1950-2050). Solar Energy 1988; 40(3): 227-35.

http://dx.doi.org/10.1016/0038-092X(88)90045-X

[7] Reda I, Andreas A. Solar position algorithm for solar radiation applications. Solar Energy 1988; 76(5): 577-89. http://dx.doi.org/10.1016/j.solener.2003.12.003

[8] Stafford B, Davis M, Chambers J, Martinez M, Sanchez D. Tracker accuracy: Field experience, analysis, and correlations with meteorological conditions. Proceedings of the 34th Photovoltaic Specialists IEEE Conference, PVSC2009, June 2009. Available from http://ieeexplore.ieee. org/xpl/login.jsp?tp=\&arnumber $=5411362 \&$ url=http $\% 3 \mathrm{~A} \% 2 \mathrm{~F}$ $\% 2$ Fieeexplore.ieee.org\%2Fxpls\%2Fabs_all.jsp\%3Farnumb er\%3D5411362 